\title{
Design of T-Shaped Micropump Based on Induced Charge Electroosmotic
}

\author{
Kai Zhang, Xiaojing Mi, and Bingying Sheng \\ Department of Mechanics, China Jiliang University, Hangzhou, Zhejiang 310018, China \\ Correspondence should be addressed to Kai Zhang; zkzb3026@gmail.com
}

Received 1 September 2013; Accepted 23 September 2013

Academic Editor: Jianzhong Lin

Copyright ( 2013 Kai Zhang et al. This is an open access article distributed under the Creative Commons Attribution License, which permits unrestricted use, distribution, and reproduction in any medium, provided the original work is properly cited.

\begin{abstract}
The fluid-driven efficiency of the micropump based on induced charge electroosmotic was studied by numerical simulation method. In this paper, we propose to make some improvement against the T-shaped piping design of micropump, and we embed a janus cylinder in the junction of the T-shaped pipe for the micropump design. We offer different voltage to the inlet of the pipe and carry out the numerical study of the fluid field induced by the cylinder, and the comparison of the velocity and flux of the outlet in different voltage as carried out. It is found that there are two symmetrical circulations around the polarizable side of the cylinder. And the comparison results show that the flow and the velocity of the outlet were increased with the increasing voltage of the entrance.
\end{abstract}

\section{Introduction}

The development of microfluidic system raises the fundamental question of how to achieve good microfluid transmission and drive results [1]. However, the phenomena of flow in microfluidic system are very different from that in the macroscopic system, which is caused by the scale effect of flow, the electric field force at the liquid/solid interface, and the coupled physics field of electric field-flow fieldtemperature field-ion movement field in the pipeline at the micron level [2]. The regular volume force (such as gravity and inertial force) is not generally important, and the electric field force becomes the leading driver of the liquid flow in microfluidic system. And the electroosmotic flow has become one of the most important ways of fluid transmission in the microfluidic chip, which does not require the machinery unit and provide the piston flow cross section.

However, there are some shortcomings for electroosmosi. For example, (1) strong electric field must be applied to the whole system to achieve the necessary field strength, generating Joule heating and raising the solution temperature, and temperature field will make feedback effect on electric field and flow field [3-5]. (2) Ac fields, which can reduce undesirable Faradaic reactions and Joule heating, produce zero time-averaged flow. Fortunately, these drawbacks do not apply to inducedcharge electroosmosis (Ramose et al. and Ajdari). Different from electroosmosis (EOF), ICEOF results from the interaction of the applied electric field and its own induced diffuse charge around immobile polarizable surfaces. The prototypical scenario involves a perfectly conducting ion-impermeable cylinder which is placed in an electrolyte solution. When an external electric field is applied, Faraday currents charge the region adjacent to its surface, thereby generating a polarized Debye layer. Simultaneously, the particle itself polarizes. The electric field exerts Lorentz body forces on its self-induced Debye cloud, thereby generating a velocity field [6].

In comparison with EOF, the velocity of ICEOF may be higher because of its nonlinear dependence on the applied electric field. Those unique characteristics may lead to new applications in microfluidics and nanofluidics. Recent research includes using ICEOF for mixing $[7,8]$ and flow regulating $[9,10]$ and promoting stirring and chaotic advection [11], the particles in Brownian motion [12], particle-wall interaction [13] and particle-particle interaction [14], nonspherical particles [15], and suspension dynamics [16].

In general, the induced charge electroosmosis (ICEOF) could be used for the design of micro-pump in microfluidic system. The impetus of this paper is to advance the understanding of induced charge electroosmosis (ICEOF) around 


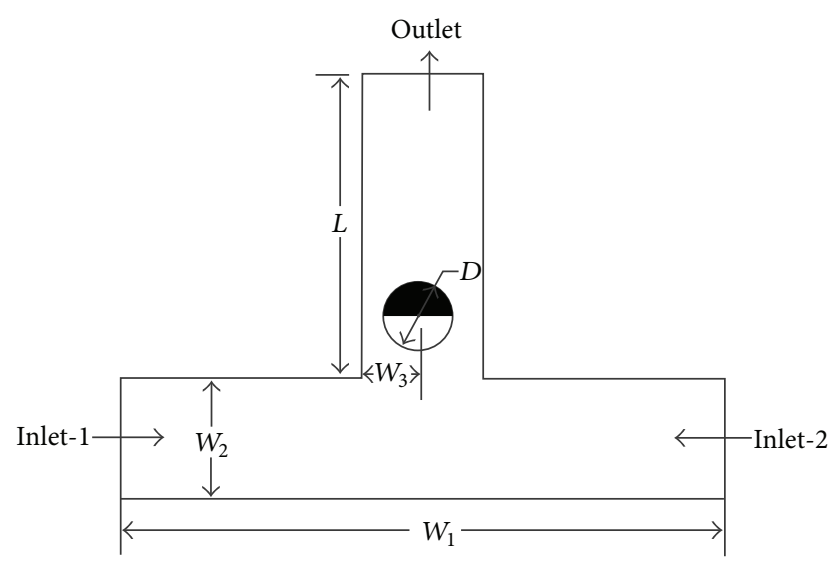

FIGURE 1: Schematic diagram of the T-shaped microchannel with Janus cylinder.

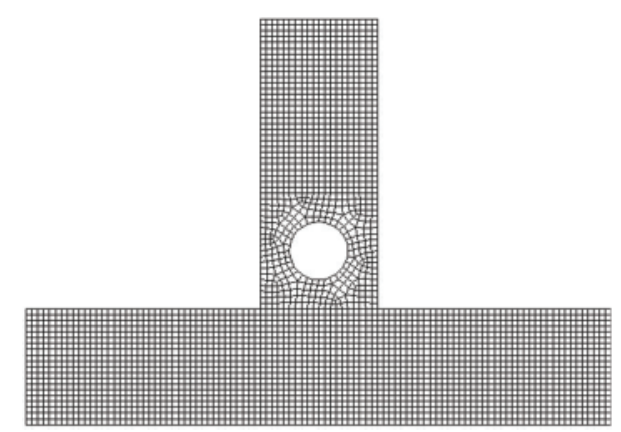

FIgURE 2: The mesh used in numerical simulation.

a Janus cylinder in a confined T-shaped microchannel, and it is mostly concerned with the design of a super efficient $\mathrm{T}$ shaped micro-pump. The relationship between fluid field and extra electric field is also studied in this research.

\section{Mathematical Model and Boundary Conditions}

2.1. Mathematical Model. The design of the T-shaped micropump with a Janus cylinder is shown in Figure 1, and the black side of the cylinder is nonpolarizable and the white side is polarizable, and Figure 2 is the mesh of the computational domain.

For the design of the T-shaped micro-pump, we study the ICEOF around the Janus cylinder, and the model parameters are shown in Table 1.

Assuming the flow is incompressible and steady and driven by the ICEOF, the momentum equation of flow can be given as

$$
\rho(\vec{V} \cdot \nabla) \vec{V}=-\nabla p+\mu \nabla^{2} \vec{V}+\vec{F}
$$

where $\vec{V}$ is velocity vector, $p$ is pressure, and $\rho$ and $\mu$ denote the density and the viscosity of the solution, respectively.

Based on the property of flow field, two velocity components are described by $u=u(x, y), v=v(x, y)$. In addition, the flow is driven by electroosmosis, and then (1) can be expressed as

$$
\rho(\vec{V} \cdot \nabla) \vec{V}=-\nabla p+\mu \nabla^{2} \vec{V}+\rho_{e} \vec{E},
$$

where $\vec{E}$ is the electric field, which is given by $\vec{E}=\nabla \Psi, \Psi$ is the electric potential, and $\rho_{e}$ is the charge density. The relation between the net charge density $\rho_{e}$ and the electrical potential $\Psi$ is shown as follows

$$
\frac{\partial^{2} \Psi}{\partial x^{2}}+\frac{\partial^{2} \Psi}{\partial y^{2}}=-\frac{\rho_{e}}{\varepsilon \varepsilon_{0}},
$$

where $\varepsilon$ is the dielectric constant of the electrolyte solution and $\varepsilon_{0}$ is the permittivity of vacuum.

In general, ion concentration is affected by both the distribution of the externally applied potential, $\varphi$, and the distribution of the potential, $\psi$, associated with the electrical double layer (with surface potential, $\zeta$ ). The overall electric potential, $\Psi$, is composed of both $\varphi$ and $\psi$. However, in general, the EDL potential distribution $\psi$ is only a small fraction of $\Psi$. Since the Debye length $\left(\lambda_{d}\right)$ is typically very small compared to the microchannel height, the ion distribution is influenced primarily by the $\zeta$ potential. It is reasonable to assume that the electric potential $\Psi$ is given by the linear superposition of the electrical double layer potential and the externally applied potential, that is, $\Psi=$ $\psi+\varphi$. Therefore, (3) can be represented as

$$
\begin{gathered}
\frac{\partial^{2} \psi}{\partial x^{2}}+\frac{\partial^{2} \psi}{\partial y^{2}}=-\frac{\rho_{e}}{\mathcal{E} \varepsilon_{0}}, \\
\nabla^{2} \phi=0,
\end{gathered}
$$

where,

$$
\rho_{e}=-2 n_{\infty} z e \sinh \left(\frac{z e \psi}{k_{b} T}\right),
$$

where $z$ is the valence of ions, $e$ is the fundamental electric charge, $n_{\infty}$ is the ionic number concentration in the bulk solution, $T$ is the absolute temperature of the solution, and $k_{b}$ is Boltzmann's constant. Taking (5) into (2) results in

$$
\rho(\vec{V} \cdot \nabla) \vec{V}=-\nabla p+\mu \nabla^{2} \vec{V}+2 n_{\infty} z e \sinh \left(\frac{z e \psi}{k_{b} T}\right) \nabla(\psi+\phi) .
$$

2.2. Boundary Conditions. For fluid flow, atmospheric pressure is specified at the inlet and outlet, and there is no slip boundary condition on the wall. For external potential, a constant value for potential is specified at the inlet and outlet, and its normal-differential value on the wall is zero. For EDL potential, its normal-differential value on the inlet and outlet is zero. Now, we will discuss the surface electric potential in detail.

Standard electric flow contains the interaction between the external electric potential and the fixed electric double layer. Thus, the electroosmotic flow velocity is linearly 

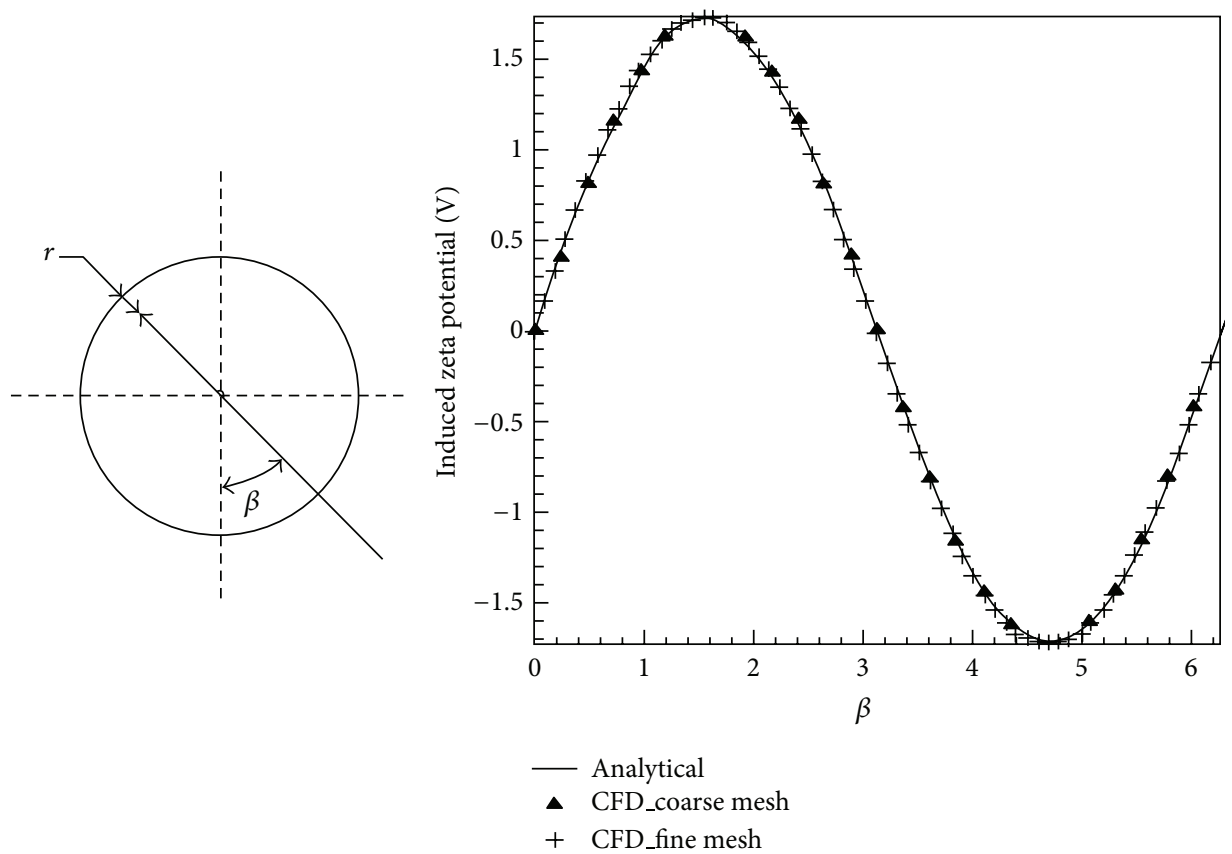

FIGURE 3: Schematic diagrams of induced zeta potential distribution on the polarizable particle's surface. $E_{0}=42.76 \mathrm{KV} / \mathrm{m}, r=20 \mu \mathrm{m}$.

TABLE 1: Model parameters.

\begin{tabular}{lcc}
\hline Parameter & Value & Description \\
\hline$W_{1}$ & $100 \mu \mathrm{m}$ & The distance between inlet-1 and inlet-2 \\
$W_{2}$ & $20 \mu \mathrm{m}$ & The width of the microchannel \\
$W_{3}$ & $10 \mu \mathrm{m}$ & The distance between cylinder center and side wall \\
$L$ & $50 \mu \mathrm{m}$ & The length of vertical pipe of T-shaped microchannel \\
$D$ & $10 \mu \mathrm{m}$ & The diameter of the Janus cylinder \\
\hline
\end{tabular}

dependent with the external electric field strength. However, when the solid surface is polarizable and conductive, the interrelation between the two will be very different. In this case, the induced zeta potential $\zeta_{i}$ is dependent with the local electric field strength $E$ and differs depending on the location of the conductive surface. In the early study, an easy correction method was proposed to achieve the numerical simulation of the zeta potential of the conductive surface induced by the local external electric potential $\varphi_{e}$. The induced zeta potential $\zeta_{i}$ is shown as

$$
\zeta_{i}=-\phi_{e}+\phi_{c}
$$

where

$$
\phi_{c}=\frac{\int_{S} \phi_{e} d A}{A}
$$

is an electric potential constant revision. Obviously, now, the induced zeta potential $\zeta_{i}$ is no longer a constant but will change with the local external electric potential.

We choose the control-volume-based method to solve the equations, and a specific discrete method is used to get the second-order accuracy. Firstly, we solve (7) and (4) to get the zeta potential and the external electric potential distribution in microchannel. Then, we solve (6) to get the flow field situation. In addition, in numerical situation, we should certificate the grid-independent to ensure the statistical independence of the calculations. As shown in Figure 2, the difference caused by the further mesh optimization above this number of grid is less than $1 \%$.

2.3. Validation. Evaluating the induced zeta potential around conducting surface is critical to calculate ICEOF, and for 2D circular cylinder, our numerical scheme has been validated by the comparison with the analytical formulation that has been derived (Bazant \& Squires [6]) as shown in

$$
\zeta=2 E_{0} r \cos \beta \text {, }
$$

where $r$ is the radius of cylinder, $E_{0}$ is the averaged electric field around cylinder, and $\beta$ is the angle as shown in Figure 3. The results show a good matching between the numerical and the analytical formulations.

\section{Results and Discussion}

The present simulation assumes that the T-shaped microchannel is made of silica glass. And it is assumed 


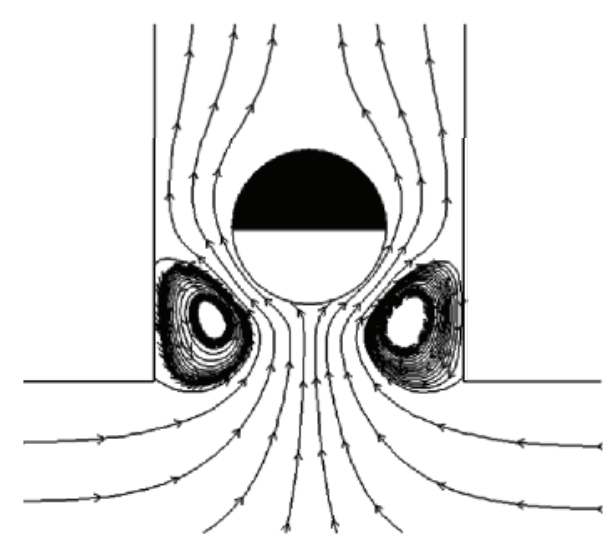

FIGURE 4: The flow diagram around the Janus cylinder when $E=$ $2 e 5 \mathrm{~V} / \mathrm{m}$.

that water-liquid is used as the working fluid and its physical properties are given by $\varepsilon=80, \varepsilon_{0}=8.85 e-12 \mathrm{CV}^{-1} \mathrm{~m}^{-1}$, $\mu=1.003 e-3 \mathrm{kgm}^{-1} \mathrm{~s}^{-1}, \rho=998.2 \mathrm{~kg} / \mathrm{m}^{3}$. All the numerical solutions presented in the following have been carefully studied such that grid-independent solutions are obtained.

In this investigation, the microchannel has an external electric potential of $\phi_{\text {out }}=0 \mathrm{~V}$, while $\phi_{\text {in }}$ is changeable from $10 \mathrm{~V}$ to $50 \mathrm{~V}$; accordingly, the electric field strength varies from $E=2 e 5 \mathrm{~V} / \mathrm{m}$ to $E=1 e 6 \mathrm{~V} / \mathrm{m}$. The zeta potential at the microchannel wall is zero, and that of the conductive surface can be obtained from (3).

As previously stated, when the conducting cylinders are immersed in the electric field, a nonuniform distribution of zeta potential will be induced on the conducting surfaces, causing a varying driving force of the electroosmotic flow. Consequently, the slipping velocity on the conducting surfaces changes with position, resulting in a nonuniform flow field. Due to the oppositely charged surfaces, flow circulations are generated near the conductive side of the embedded cylinder.

Here, we offer the flow diagram around the Janus cylinder when $E=2 e 5 \mathrm{~V} / \mathrm{m}$ and $E=1 e 6 \mathrm{~V} / \mathrm{m}$, as shown in Figures 4 and 5 , respectively. We can see that consistent with the theoretical analysis, there exist 2 symmetrical flow circulations around the conductive side of the cylinder. In addition, we can find that the flow circulations will become smaller significantly and approach to the pipe wall but still be symmetrical.

For better study of the relationship between external electric field strength and the T-shaped pump driven efficacy, we make numerical simulation when $\phi_{\text {in }}=10 \mathrm{~V}, 20 \mathrm{~V}, 30 \mathrm{~V}$, $40 \mathrm{~V}$, and $50 \mathrm{~V}$, respectively. As shown in Figure 6, at the outlet of the microchannel, the velocity gradient increases with the increasing of the electrical field strength, which means that the driven efficacy of the T-shaped micro-pump increases with the increasing of the electrical field. For better description of the driven efficiency, we figure out the average velocity magnitude $\bar{V}$ at the outlet and offer the corresponding flux $Q=\bar{V} * W_{3}$. As shown in Figure 7, we

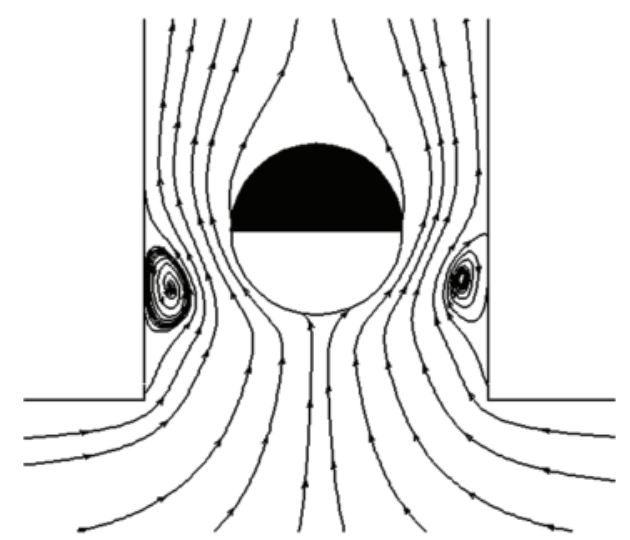

Figure 5: The flow diagram around the Janus cylinder when $E=$ $2 e 5 \mathrm{~V} / \mathrm{m}$.

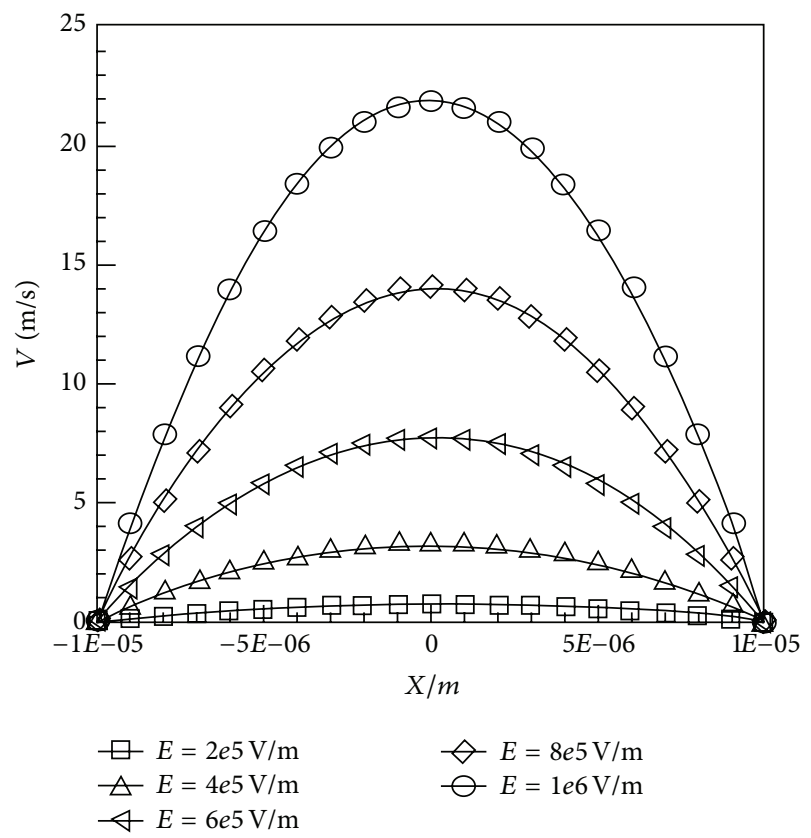

FIGURE 6: The velocity magnitude profiles under different applied electric field.

can quantitatively see that the variation range of the T-shaped micro-pump flux is from $12.41 \mathrm{~mL} / \mathrm{s}$ to $348 \mathrm{~mL} / \mathrm{s}$.

In summary, the T-shaped micro pump embedded a Janus cylinder, and we propose in this paper that good fluid-driven efficiency can be obtained under small external electric potential, which is of practical value.

\section{Conclusions}

In this paper, we offer a design of T-shaped micro-pump that embedded a Janus cylinder. We carry out the numerical study of the fluid field induced by the cylinder and make comparison between the velocity magnitude and flux of the outlet in different voltage. It is found that there are two symmetrical flow circulations around the polarizable side 


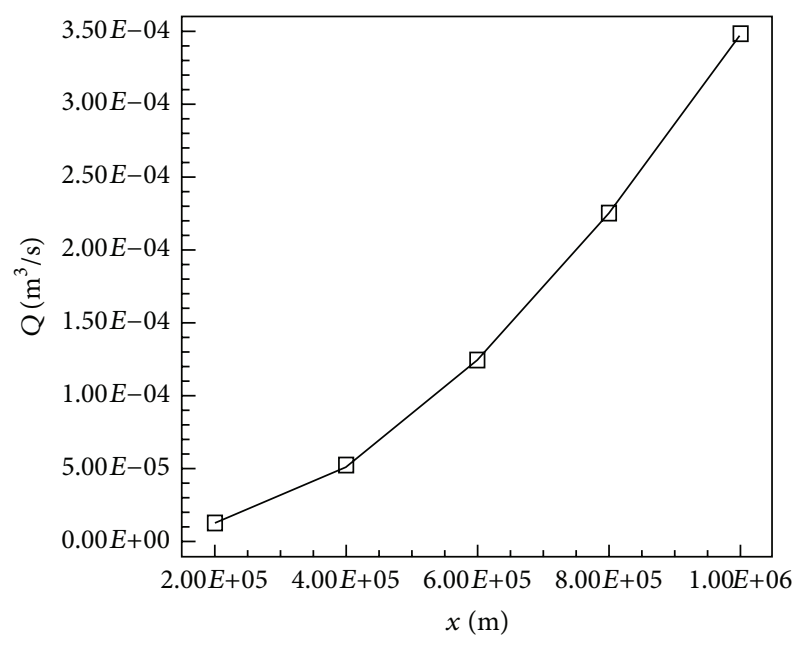

Figure 7: The flux at the outlet of the microchannel under different applied electric field.

of the Janus cylinder, and they can be used to improve the driven efficiency of the pump. The dependence of the driven efficiency on the electric field is also predicted. The conclusions above can be utilized for the optimization of the design of microfluidic devices.

\section{Acknowledgments}

The authors are extremely grateful to the editor and the anonymous reviewers for their constructive and valuable comments, which have contributed much to the improvement of this paper. Also, the authors gratefully acknowledge the financial support from the National Natural Science Foundation of China with Grant no. 10902105 and the Natural Science Foundation of Zhejiang Province with Grant no. Y6090406/2010R10014.

\section{References}

[1] J. Clayton, "Go with the microflow," Nature Methods, vol. 2, no. 8, pp. 621-627, 2005.

[2] G. H. Mohamed, The MEMS Handbook, CRC Press, New York, NY, USA, 1999.

[3] K. Zhang and J. Lin, "The effect of temperature distribution on the mass species transport in micro-channels driven by electroosmosis," in Proceedings of the Coference of Global Chinese Scholars on Hydynamics (CCSH '06), pp. 65-70, 2006.

[4] E. Grushka, R. M. McCormick, and J. J. Kirkland, "Effect of temperature gradients on the efficiency of capillary zone electrophoresis separations," Analytical Chemistry, vol. 61, no. 3, pp. 241-246, 1989.

[5] A. E. Jones and E. Grushka, "Nature of temperature gradients in capillary zone electrophoresis," Journal of Chromatography A, vol. 466, pp. 219-225, 1989.

[6] M. Z. Bazant and T. M. Squires, "Induced-charge electrokinetic phenomena: theory and microfluidic applications," Physical Review Letters, vol. 92, no. 6, Article ID 066101, 2004.

[7] Z. Wu and D. Li, "Mixing and flow regulating by induced-charge electrokinetic flow in a microchannel with a pair of conducting triangle hurdles," Microfluidics and Nanofluidics, vol. 5, no. 1, pp. 65-76, 2008.

[8] J.-Z. Lin, K. Zhang, and H.-J. Li, "Study on the mixing of fluid in curved microchannels with heterogeneous surface potentials," Chinese Physics, vol. 15, no. 11, pp. 2688-2696, 2006.

[9] K. Zhang, J.-Z. Lin, and Z.-H. Li, "Research on diffusion in micro-channel flow driven by electroosmosis," Applied Mathematics and Mechanics (English Edition), vol. 27, no. 5, pp. 575582, 2006.

[10] Z.-H. Li, J.-Z. Lin, and D.-M. Nie, "New approach to minimize dispersion induced by turn in capillary electrophoresis channel flows," Applied Mathematics and Mechanics (English Edition), vol. 26, no. 6, pp. 685-690, 2005.

[11] H. Zhao and H. H. Bau, "Microfluidic chaotic stirrer utilizing induced-charge electro-osmosis," Physical Review E, vol. 75, no. 6, Article ID 066217, 2007.

[12] M. Yu, J. Lin, and T. Chan, "A new moment method for solving the coagulation equation for particles in Brownian motion," Aerosol Science and Technology, vol. 42, no. 9, pp. 705-713, 2008.

[13] Z. Wu and D. Li, "Induced-charge electrophoretic motion of ideally polarizable particles," Electrochimica Acta, vol. 54, no. 15, pp. 3960-3967, 2009.

[14] D. Saintillan, "Nonlinear interactions in electrophoresis of ideally polarizable particles," Physics of Fluids, vol. 20, no. 6, Article ID 067104, 10 pages, 2008.

[15] D. Saintillan, E. S. G. Shaqfeh, and E. Darve, "The growth of concentration fluctuations in dilute dispersions of orientable and deformable particles under sedimentation," Journal of Fluid Mechanics, vol. 553, pp. 347-388, 2006.

[16] E. Yariv, "Slender-body approximations for electro-phoresis and electro-rotation of polarizable particles," Journal of Fluid Mechanics, vol. 613, pp. 85-94, 2008. 


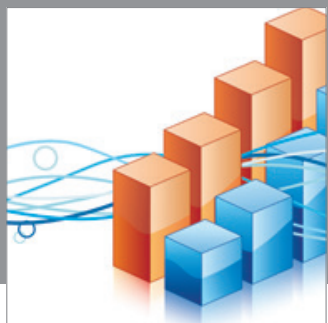

Advances in

Operations Research

mansans

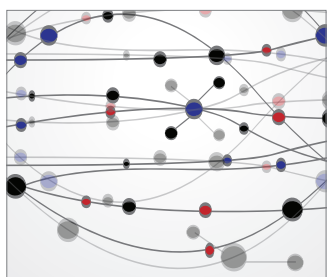

The Scientific World Journal
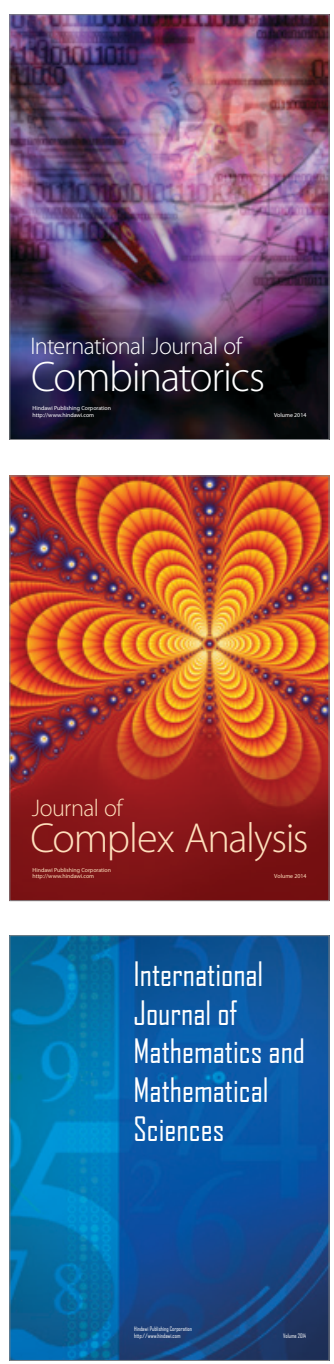
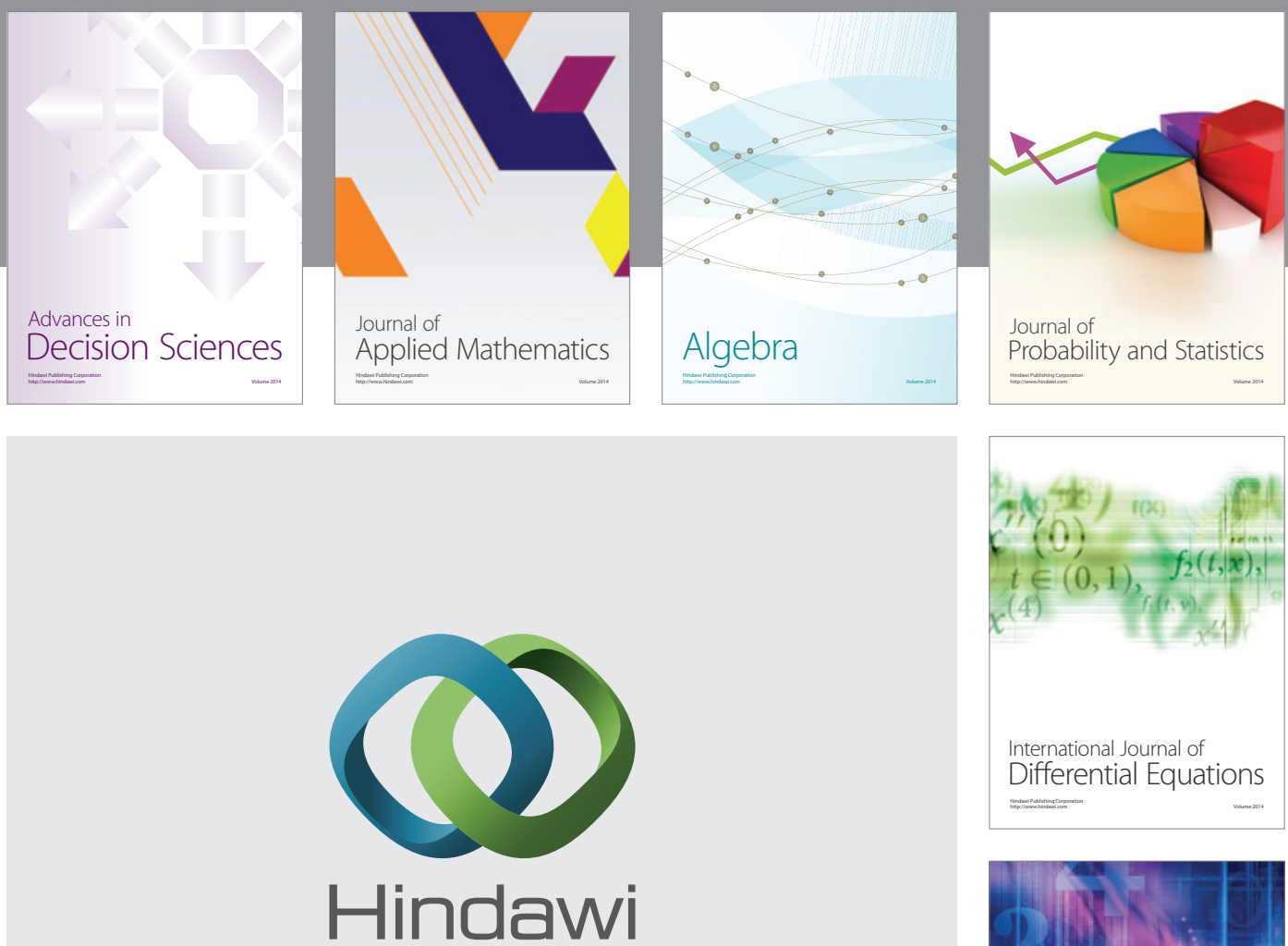

Submit your manuscripts at http://www.hindawi.com
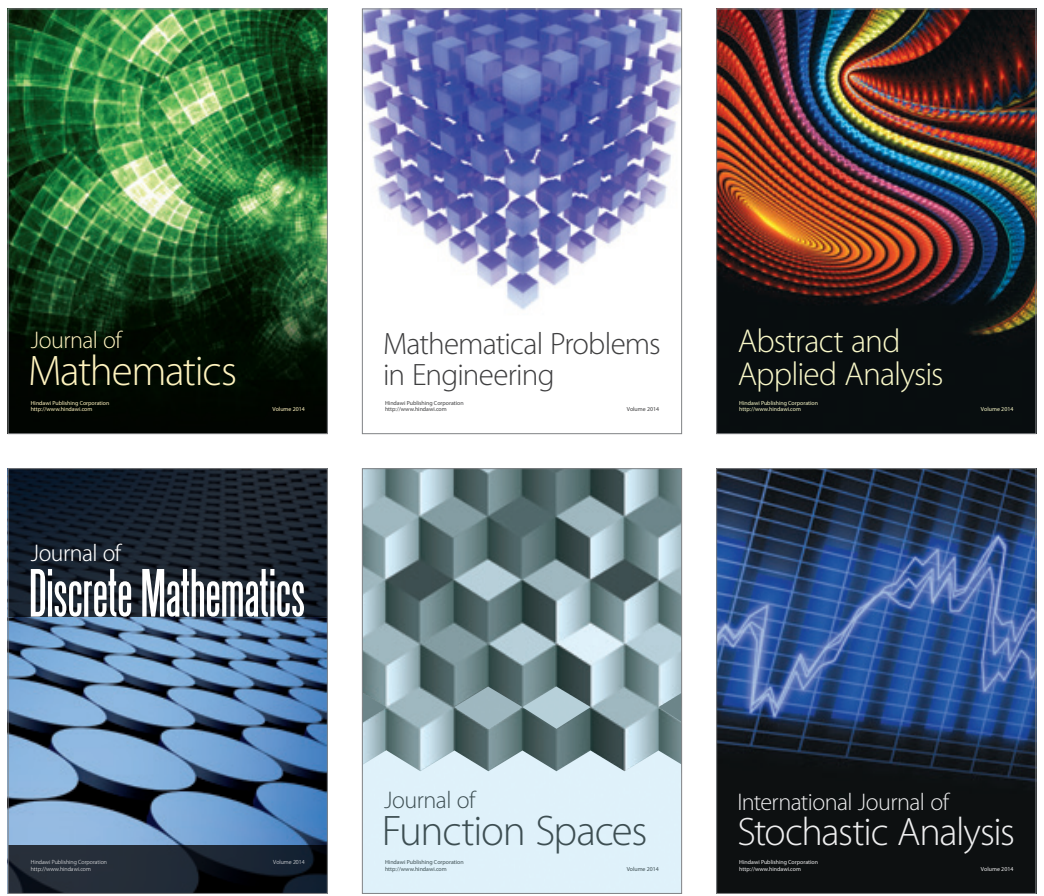

Journal of

Function Spaces

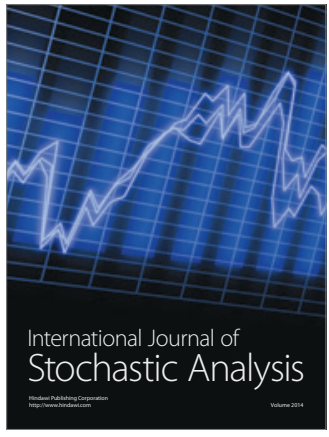

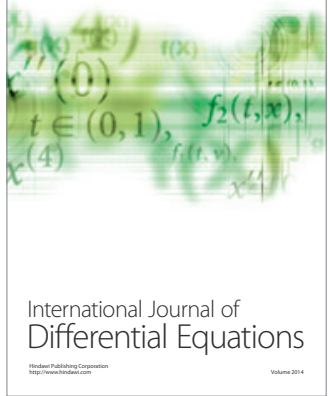
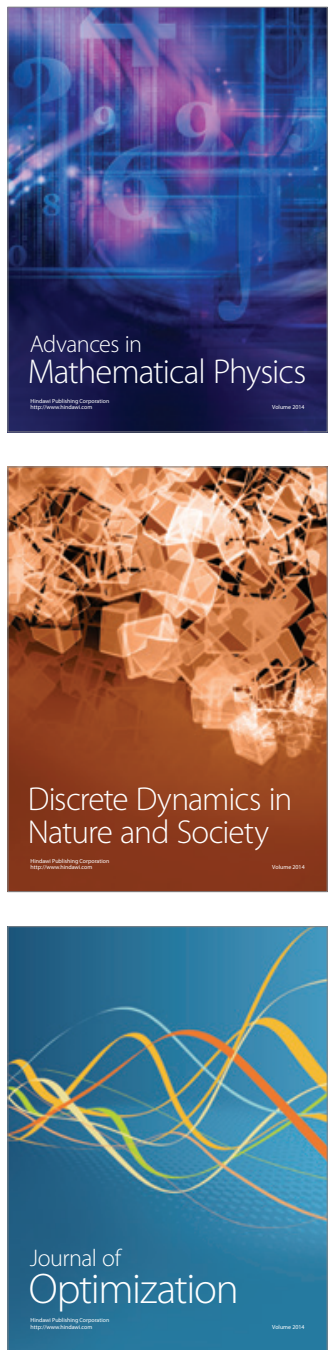